\title{
AOR
}

Selected Papers of \#AoIR2018:

The $19^{\text {th }}$ Annual Conference of the

Association of Internet Researchers

Montréal, Canada / 10-13 October 2018

\section{DISTRIBUTING SERVICES THROUGH THE CLOUD INFRASTRUCTURE: THE CASE OF A NORWEGIAN SOFTWARE FIRM}

\author{
Christian S. Ritter \\ Norwegian University of Science and Technology
}

\section{Introduction}

This paper outlines an ethnographic approach to the study of cloud infrastructures. Analyzing evidence from ethnographic fieldwork in a Norwegian software firm, I examine how the everyday practices of programmers developing industrial software configure socio-technical assemblages within the global oil and gas industry. The Norwegian software firm delivers business intelligence software to oil and gas corporations. Generating insightful dashboards, the software assists engineers in their decision-making on technical equipment and operational risks. The main purpose of this paper is to advance the understanding of the dynamic interplay between human and non-human actors in socio-technical assemblages. I initially negotiated access to field sites and ethnographic data by attending industry events. However, a three-month secondment in the firm under investigation was invaluable for the collection of data since I was granted access to cloud-based software, developer platforms, and internal meetings. The data set is comprised of observation records from the secondment, 30 indepth interviews with employees, event organizers, and industry experts, as well as organizational materials, including company blogs, reports on industry events and internal documents. Based on an extensive assessment of the evidence collected, I argue that programmers of the industrial software embody the capacity to scale sociotechnical assemblages by building application programming interfaces (APIs) that control the access for communities of practice to data stored in the cloud infrastructure.

\section{Material worlds within cloud infrastructures}

The conceptual frame that gives direction to this study combines a materialistic take on internet infrastructures with a renewed understanding of socio-technical assemblages. Infrastructures can be seen as built networks that facilitate the flows of goods, services,

Suggested Citation (APA): Ritter, C. (2018, October 10-13). Distributing services through the cloud infrastructure: The case of a Norwegian software firm. Paper presented at AolR 2018: The 19 ${ }^{\text {th }}$ Annual Conference of the Association of Internet Researchers. Montréal, Canada: AolR. Retrieved from http://spir.aoir.org. 
people, or ideas and allow for symbolic exchange over space (e.g. Larkin 2013). The sociality and everyday practices occurring within internet infrastructures are embedded in material worlds, including complex orders and relations among artefacts themselves and their relationships to persons (Horst \& Miller, 2012). Digital infrastructures are material entities in the sense that they carry the symbolic meanings persons exchange through the internet. Symbolic meanings are actualized through the material worlds of artefacts. The cloud infrastructure constitutes socio-technical assemblages which are grounded in material worlds, including data centers, open office spaces, computer screens, and the affordances of interfaces. The software of the firm under investigation generates one of these socio-technical assemblages in which various professional groups can meaningfully interact. Assemblages are basically configurations of the relations between parts and wholes. They involve multiple collections of persons and artefacts. Consisting of various components, socio-technical assemblages are enacted through everyday practices tied to technological configurations (Dodson 2014).

The main components of the socio-technical assemblage that the software under investigation generates are source code, cloud technologies, digital services, and interfaces. The source code is the initial version of a software written by a human (LIP 2018). Cloud service providers, such as Amazon Web Services and Microsoft Azure, provide on-demand services that enable programmers to store large-sized databases and to build software within the cloud infrastructures. Digital services, such as access to databases and dashboards showing business insights, circulate through the sociotechnical assemblage. The interface is a central component of cloud infrastructures. Interfaces can be broadly defined as shared boundaries cross which symbolic meanings can be exchanged. They serve as meeting points where components of systems interact. Furthermore, interfaces are autonomous zones of activities and points of transition between different mediatic layers within a nested system (Galloway 2013). Ethnographic researchers can observe everyday practices in interfaces to explore relations among humans and between humans and artefacts.

\section{Inside a socio-technical assemblage}

This investigation identifies various actors in the socio-technical assemblage that the software under investigation generates. Shadowing the work routines of consultants and programmers in the Norwegian software firm, I traced the circulation of digital services within the socio-technical assemblage. Two ethnographic cases illustrate how digital services are distributed through the cloud infrastructures. Both cases describe interfaces as crucial components of the socio-technical assemblage. These artefacts of the assemblage are actors with substantial agency.

The first case traces an 'issue' through various interfaces of the cloud infrastructure. A text field in a popup window displayed the wrong message, which was spotted on a computer screen in Houston, USA. The users of the software reported this bug to a consultant during a support call. The consultant added an 'issue' describing the flaw in the graphical user interface (GUI) to the backlog of the developer platform. In a grooming session, which was held in the following week, the product owner and scrum master prioritized the issues that needed to be completed prior to the next release. The flaw identified in Houston was among the urgent issues. After the release of the new version of the software, the Houston-based users could experience the newly designed 
affordances of the software on their computer screen. The observable everyday practices of human actors, such as consultants and software users, indicate that the cloud infrastructure enables a transnational built network connecting the transnationally dispersed material worlds of office spaces.

The second case that illustrates the circulation of digital services through cloud infrastructures deals with the Application Programming Interfaces (APIs) of the software. An employee of a client company requested access to a database of the software during a further support call with a consultant. After a discussion between the consultant and a programmer in an open office of the firm, the client was provided with an access key and could call the API to retrieve data about the technical equipment used for the extraction of oil and gas. The analysis of the access rights to databases of the software reveals the programmed hierarchy that structures who can access and analyze the data for specific pieces of equipment, specific operators, or the entire industry. Both cases indicate that interfaces play a central role in the circulation of digital services and the workflows of software development.

\section{Conclusion}

Cloud infrastructures make the collaboration among firms in different regions possible. The programmers of the cloud-based software steer flows of business insights through a socio-technical assemblage by building APIs that include and exclude members in accordance with subscription rates to digital services. Based on the evidence studied, I suggest that programmers can scale the socio-technical assemblage by writing scripts that organize the membership hierarchies within the APIs of industrial software. In addition to the agency that programmers exert, the circulation of the digital service through the socio-technical assemblage is configured by the affordances of various artefacts. For example, cloud services and backlogs structure the everyday practices of software development. The findings of the study imply that cloud technologies enable new infrastructures connecting human and non-human actors in ever-greater transnational settings.

\section{References}

Dodson, J. (2013). Suburbia under an energy transition: A socio-technical perspective. Urban Studies 51(7), 1487-1505.

Galloway, A. (2013). The Interface Effect. Malden, MA: Polity.

Horst, H. \& Miller, D. (2012). The digital and the human: a prospectus for digital anthropology. In Horst, H. \& Miller, D. (ed.), Digital Anthropology. (pp. 3-38). London and New York: Berg.

Larkin, B. (2013). The politics and poetics of infrastructure. Annual Review of Anthropology 42(1), 327-343. 
The Linux Information Project (2018). Source Code Definition.

http://www.linfo.org/source_code.html. Accessed: 28/09/2018. 